\title{
Sonderheft zum
}

\section{Deutschen Orthopädenkongreß 1988 \\ vom 16.-20. September in Saarbrücken}

\section{Editorial}

Der Deutsche Orthopädenkongreß 1988 war der erste Gemeinschaftskongreß der Deutschen Gesellschaft für Orthopädie und Traumatologie mit dem Berufsverband der Ärzte für Orthopädie. Konzept und Organisation dieses Kongresses mußten in vielen Bereichen von den eingefahrenen Strukturen früherer Tagungen abweichen, so auch bei der Veröffentlichung der Vorträge.

Wie seit der 72. Tagung der DGOT (1985, Frankfurt) üblich, wurden die Abstrakte aller angenommenen Vorträge in Heft 3/88 des Mitteilungsblattes (Demeter Verlag) publiziert. Die Gemeinschaftsveranstaltungen mit dem Berufsverband, das 2. und 3. Symposium mit der Thematik Probleme der Brust- und Lendenwirbelsäule, sind als 20. Band der vom Berufsverband herausgegebenen Reihe Praktische Orthopädie veröffentlicht. Das vorliegende Sonderheft enthält die Vorträge der öffentlichen Sitzungen des Arbeitskreises (AK) Knochentumoren, des AK Dokumentation und Statistik sowie des AK Krankenhaushygiene. Die Gliederung des Heftes entspricht dieser Reihenfolge.

Die Vorträge des AK Knochentumoren geben einen hervorragenden Überblick über die aktuellen Therapiekonzepte und deren Erfolge bei intraläsionaler Tumorresektion, vorwiegend bei Riesenzelltumoren und Chondromen. Die im Arbeitskreis zusammengetragenen Erfahrungen sind eine wichtige Orientierungshilfe für die Kliniken, die weniger häufig mit Knochentumoren und ihrer Problematik konfrontiert werden. Die Veröffentlichung der Vorträge des AK Knochentumoren ist um so wichtiger, als diese Thematik in den letzten Jahren bei den Kongreßveranstaltungen der DGOT wenig vertreten war.

Der AK Dokumentation und Statistik befaßt sich mit der Durchführung der Krankenhausstatistik mit Hilfe von EDV-Systemen und mit den Erfahrungen in der Anwendung der Bundespflegesatzverordnung von 1985/86. Dokumentation und Statistik gewinnen aus haftungs- und verwaltungsrechtlichen Gründen in Klinik und Praxis zunehmend an Bedeutung. Die fachlichen Kenntnisse und geräteabhängigen Voraussetzungen für die erforderlichen Maßnahmen sind aber bisher nur an den wenigsten Kliniken im notwendigen Umfange vorhanden. Die Arbeiten des Arbeitskreises zeigen, daß auch

Z. Orthop. 127 (1989) 375

(C) 1989 F. Enke Verlag Stuttgart beim Vorliegen dieser Voraussetzungen in der praktischen Durchführung der Statistik und ihrer Dokumentation Lernprozesse $\mathrm{zu}$ absolvieren sind. Im orthopädischen Bereich ist dieser Vortragskomplex die erste zusammenfassende Darstellung dieser Thematik. Sie bietet dem Interessierten, aber noch Unkundigen Material und Möglichkeit zum einführenden Studium mit nützlichen Literaturhinweisen. In jeder Klinik und ärztlichen Praxis, ganz gleich, ob sie schon Dokumentation und Statistik mit den modernen Möglichkeiten der EDV betreibt oder damit erst beginnen will, sollten die vorliegenden Arbeiten bekannt sein.

Der AK Krankenhaushygiene gibt mit seinen Vorträgen einen hervorragenden Überblick über die komplexe Hygiene-Problematik im Krankenhaus, die schon realisierbaren Lösungsstrategien und über die noch offenen Fragen. Auch dieser Vortragskomplex ist in der Geschlossenheit der Darstellung zu dieser Thematik im orthopädischen Bereich erstmalig.

Aufgrund der Bedeutung der drei Themenkomplexe dieses Sonderheftes bedaure ich es sehr, $\mathrm{da} \beta$ ich wegen der vorgegebenen Umfangsbegrenzung des Heftes gezwungen war, einige Vorträge zu kürzen, vorwiegend durch Streichung von Abbildungen und Tabellen, in Einzelfällen auch durch Textkürzungen. Dabei habe ich aber sehr sorgfältig darauf geachtet, daß die wesentlichen Aussagen der Vorträge erhalten blieben.

In Anerkennung der Bedeutung des $\mathrm{Hy}$ giene-Themas hat sich der Enke-Verlag jedoch entschlossen, die aktuellen Probleme der Krankenhaushygiene einschließlich der AIDS-Problematik als Buch unter dem Titel Krankenhaushygiene in Orthopädie und Traumatologie zu veröffentlichen. Dieses Buch wird auch die Kongreßvorträge des AK Krankenhaushygiene in ungekürzter Form mit allen Abbildungen und Tabellen enthalten.

An dieser Stelle möchte ich allen Autoren für ihre Mitarbeit danken. Besonderen Dank schulde ich den Leitern der Arbeitskreise, Herrn Prof. Dr. R. Kotz, AK-Knochentumoren, Herrn Dr. Th. Winter, AK-Dokumentation und Statistik und Herrn Prof. Dr. A. Härle, AK-Krankenhaushygiene, für die Auswahl der Thematik und der Referenten sowie für die Vororganisation der öffentlichen AK-Sitzungen beim Deutschen Orthopädenkongreß 1988. 